\title{
Hacia la escuela inclusiva en Perú. Estudio sobre la aplicación de una propuesta de trabajo de las Ciencias en Educación Básica Regular
}

\author{
Esther Cascarosa Salillas. Universidad de Zaragoza \\ Marta Marco. Colegio Santa Ana, Zaragoza \\ Beatriz Mazas Gil. Universidad de Zaragoza \\ Recepción: 02.09.2019 | Aceptado: 15.10.2019 \\ Correspondencia a través de ORCID: Esther Cascarosa \\ iD 0000-0002-3696-7673 \\ Citar: Cascarosa, E., Marco, M. y Mazas, B. (2019). Hacia la escuela inclusiva en Perú. Estudio sobre la \\ aplicación de una propuesta de trabajo de las Ciencias en Educación Básica Regular. REIDOCREA, 8, 221- \\ 230.
}

Agradecimientos: Los autores agradecen al proyecto MINECO EDU2016-76743-P su apoyo en el desarrollo de este trabajo, al grupo BEAGLE financiado por el Gobierno de Aragón (S27-17R) y cofinanciado con Feder 2014-2020, y al Instituto IUCA, ambos de la Universidad de Zaragoza.

Resumen: Para que una escuela se llame inclusiva debe contemplar a todos los factores que sobre ella tienen impacto, en especial a los alumnos. Una escuela será inclusiva hacia los alumnos cuando sea capaz de considerar las necesidades de cada uno de estos. Estas necesidades no tienen por qué ser solo conceptuales, sino también actitudinales o procedimentales. Tomando lo anterior como base, se diseñó y se desarrolló una propuesta para trabajar las Ciencias Naturales en una clase de Educación Básica Regular en Perú. La propuesta se implementó a través de siete actividades para trabajar el aparato locomotor del cuerpo humano de manera práctica, con elementos cercanos al alumno y de forma cooperativa. También se atendieron las necesidades personales de cada alumno, adaptando las actividades de manera personalizada. Aunque motivados, los alumnos muestran una resistencia inicial al cambio metodológico debido a su hábito de escuchar y repetir la lección.

Palabras clave: Ciencias de la naturaleza | Educación inclusiva

Towards inclusive school in Peru. Study on the application of a proposal to work Sciences in Regular Basic Education

Abstract: So a school called inclusive should consider all factors have impact on it, especially to students. A school will be inclusive to the students when it is able to consider the needs of each of these. These needs do not have to be only conceptual, but also attitudinal or procedural. Taking the above as a basis, it was designed and a proposal was developed to work the natural sciences in a class of Regular Basic Education in Peru. The proposal was implemented through seven activities to work the musculoskeletal system of the human body in a practical way, with elements close to the student and in a cooperatively way. Also discussed the personal needs of each student, adapting the activities in a personalized way. Although motivated, students show an initial resistance to methodological change due to their habit of listening and repeating the lesson.

Keywords: Natural Sciences | Inclusive Education

\section{Introducción}

Hablar de escuela inclusiva implica hablar de un centro educativo donde todos los agentes participan de manera activa (Calvo, Verdugo y Amor, 2016). En particular, los alumnos deben tener un papel protagonista en las aulas y actuar de constructores de su propio aprendizaje. En ocasiones, se olvida la importancia del papel activo que debe tener el alumno y la función de éste queda limitada a mero receptor de la información transmitida por el maestro. Las aulas deberían ser lugares en los que se propicie que el estudiante vaya más allá de la simple memorización de hechos, enseñándoles a tomar la iniciativa y ser responsables de su propio aprendizaje (Cuevas, Lee, Hart y Deaktor, 2005). Parece fundamental que las metodologías educativas aplicadas en el aula consideren al alumno como el centro del proceso educativo y además sean variadas para poder conseguir una educación inclusiva de todos ellos. Hoy en día, es sabido que 
no todos los alumnos aprenden de la misma manera ni al mismo ritmo (Altun y Yazici, 2010), por lo que, si se quiere incluir en el proceso educativo a todos, deben atenderse las necesidades de cada uno de ellos, aplicando metodologías activas y utilizando materiales alternativos al libro de texto. Según el estudio realizado por García y Martínez (2001) solo el $20 \%$ de los maestros utilizaban materiales distintos al libro de texto. Esto en parte es debido a la falta de formación específica de los maestros (Kozulín, 2000).

En el caso particular del proceso de enseñanza-aprendizaje de las ciencias son muchos los autores que recomiendan partir de los elementos del entorno del alumno para construir los conceptos en base a lo conocido (Harlen, 2015). Otra metodología probada como efectiva para lograr un aprendizaje adecuado de las ciencias consiste en partir de la práctica para pensar e interpretar desde un marco teórico cada vez más abstracto, llegando a conclusiones a partir de dicha práctica (Izquierdo, Sanmartí y Espinet, 1999). A través de la indagación se puede mejorar la adquisición de los conociาmientos y destrezas propias de las ciencias e incrementar el interés del alumnado hacia esta área de conocimiento. La indagación se asocia también con el desarrollo de la creatividad, siendo esta una de las características principales de la investigación científica (Tanggaard, 2014). Un elemento crucial para que la indagación tenga éxito es el reconocimiento de la ciencia como un proceso activo que necesita del razonamiento, la observación y la experimentación, y no como una colección de hechos que han de ser memorizados por el estudiante (Lena, 2011).

A pesar de todo lo expuesto, históricamente la manera de aprender ciencias ha sido a través de clases magistrales sobre diferentes contenidos conceptuales en lugar de por medio de propuestas prácticas (Cortes et al., 2012). Trabajando las ciencias de manera memorística, no se consigue una inclusión efectiva de los alumnos, ya que no todos son capaces de utilizar la memoria por encima de otras destrezas. Y en ese sentido, se está dejando de lado el desarrollo de las competencias de éstos alumnos. Además de esto, no introducir actividades con las que los alumnos puedan dar respuesta a los fenómenos que les rodean, conduce a una baja motivación por las ciencias. Un ejemplo recogido por George (2006) muestra los resultados de estudios realizados con adolescentes donde se concluye que estos tienen una baja autoconfianza en el aprendizaje de las ciencias y bajo interés por éstas. Este desinterés y falta de motivación desemboca en una tendencia descendente en las vocaciones científicas futuras (Barmby, Kind y Jones, 2008). Si estos aspectos no se tienen en cuenta, y por lo tanto no se trabajan, la consecuencia es que muchos alumnos, no solo no disfrutan de la ciencia, sino que terminar por quedar fuera del sistema educativo y ni siquiera habrán conseguido una adecuada alfabetización científica, deseable para toda la ciudadanía. Según Solbes, Monstserrat y Furió (2007), en la enseñanza actual de las ciencias, mayoritariamente no se está teniendo en cuenta esa falta de motivación del alumnado. La autoconfianza y la motivación son dos de los aspectos más vinculados al rendimiento escolar en ciencias (Gil-Flores, 2012). Por lo tanto, parece necesario buscar una manera de contemplar estos aspectos. Por otro lado, no hay que olvidarse del carácter socializador de la escuela, muchas veces conseguido a base de aprendizaje colaborativo con interdependencias positivas para el logro de metas comunes (Marín, Negre y Pérez, 2014).

Teniendo en cuenta todo lo anterior, el objetivo de este trabajo ha sido diseñar y desarrollar una propuesta didáctica para trabajar las ciencias en el aula de manera colaborativa. Se han analizado los resultados recogidos y a través de ellos se ha evaluado si la inclusión de todos los alumnos ha sido efectiva.

\section{Métodos}


El presente estudio se ha desarrollado en una clase de tercer ciclo de Educación Básica Regular, en $2^{\circ}$ curso de Educación Primaria del centro San Juan Masía, ubicado en el Caserío de Puplán, en Mórrope localizado en la provincia de Lambayeque, en Perú. Se trata de un centro rural tipo de la zona, donde el nivel socioeconómico de la comunidad es bajo. Gran parte de las familias no poseen conocimientos académicos suficientes para poder ayudar a los alumnos, por esta razón éstos deben adquirir sus conocimientos científicos en la escuela.

En el aula donde se ha aplicado esta propuesta hay trece alumnos, ocho de los cuales son chicos y nueve chicas. De éstas, hay tres con dificultades de aprendizaje basadas en problemas de lecto-escritura. Estas alumnas arrastran un importante retraso curricular y se encuentran desmotivadas hacia el aprendizaje escolar en general y hacia el de ciencias en particular. Por el contrario, hay otras cuatro alumnas que destacan por sus conocimientos. En general, todos los alumnos de la clase necesitan reconocimiento positivo a su labor escolar como motivación extrínseca. Habitualmente en el aula se utiliza la metodología tradicional basada en la memorización de contenidos. A partir de este contexto, se pensó en introducir una propuesta para trabajar las ciencias basada en hechos que los alumnos manejaran en su día a día, situaciones o fenómenos relacionados con el campo y el cultivo, o la ganadería u otros recursos que se pueden utilizar como punto de anclaje para construir su propio aprendizaje. Como dice Gallegos (2001): hay que tener en cuenta y comprender la actividad práctica como transformadora de la naturaleza social, para ello hay que estudiar el aprendizaje y participación de los niños indígenas en las tareas que realizan fuera de la escuela. Se intenta en todo momento que los alumnos sean capaces de elaborar ellos mismos sus aprendizajes con lo que pueden trabajar y ver en su día a día como el contacto con la chacra (campo) o con los diferentes animales a los que deben cuidar, ya que son el sustento de su familia.

La propuesta de actividad que aquí se desarrolla parte de la investigación previa sobre el estudio de vocaciones científicas el contexto comentado (Cascarosa y Marco, 2018). El presente estudio tuvo por objetivo que los alumnos aprendieran por medio de la práctica y con situaciones de la vida cotidiana porque que es importante que los niños conozcan las causas de lo que ven y cómo se producen fenómenos que ellos mismos llevan a cabo, como por ejemplo el movimiento de sus cuerpos. Además de la adquisición de conceptos es imprescindible el desarrollo de actitudes, y trabajar así ayuda a conseguirlo. En base a todo esto, se diseñó e implementó una propuesta de intervención sobre el aparato locomotor del cuerpo humano, utilizando sus propios cuerpos para contextualizar el tema y facilitar su comprensión, además de la interdisciplinariedad y el uso de la música para la introducción del tema. La propuesta de trabajo se desarrolló a través de siete actividades concretas, y con cada una de ellas se perseguía un objetivo específico. Para llevarlas a cabo se preparó material particular adaptándolo a las características de los alumnos de la clase. En la Tabla 1 se recogen los contenidos y los objetivos de cada actividad.

\begin{tabular}{|cl|l|l|}
\hline \multicolumn{4}{|c|}{ Tabla 1: Descripción de las actividades desarrolladas en la clase. } \\
\hline \multicolumn{2}{|c|}{ Actividad } & \multicolumn{1}{|c|}{ Contenido } & \multicolumn{1}{c|}{ Objetivos } \\
\hline 1. & Lluvia de ideas & ¿Cómo y por qué nos movemos? & Conocer ideas previas e introducir el tema \\
\hline 2. & Canción & $\begin{array}{l}\text { Palabras relacionadas con el aparato } \\
\text { locomotor }\end{array}$ & Motivar y conocer ideas previas \\
\hline 3. & Vídeo + esquema & Partes y funciones del aparato locomotor & $\begin{array}{l}\text { Identificar los conceptos clave y } \\
\text { esquematizarlos }\end{array}$ \\
\hline 4. & Sopa de letras & $\begin{array}{l}\text { Identificación y ubicación de partes del } \\
\text { aparato locomotor }\end{array}$ & Evaluar formativa de conocimientos \\
\hline 5. & Juego de tarjetas & Ubicación las partes en un esqueleto & Cooperar grupalmente \\
\hline 6. & $\begin{array}{l}\text { Identifica en tu } \\
\text { cuerpo }\end{array}$ & $\begin{array}{l}\text { Partes del aparato locomotor del cuerpo } \\
\text { humano }\end{array}$ & $\begin{array}{l}\text { Utilizar el propio cuerpo como herramienta de } \\
\text { aprendizaje }\end{array}$ \\
\hline 7. & Ficha & Partes y funciones del aparato locomotor & Evaluación sumativa \\
\hline
\end{tabular}


Con la primera actividad se introdujo el tema y se indagó en las ideas previas y concepciones alternativas que los alumnos tenían sobre el movimiento del cuerpo, los músculos y los huesos. Para la segunda actividad, se sustituyeron palabras de una canción conocida para los alumnos, introduciendo vocabulario relativo al aparato locomotor (ver Figura 1). Al escuchar la canción, la maestra fue realizando las acciones que se describen en ella y animando a los alumnos a que hicieran lo mismo. Al finalizar, se leyó la letra de la canción en voz alta, intentado identificar las palabras que les eran desconocidas.

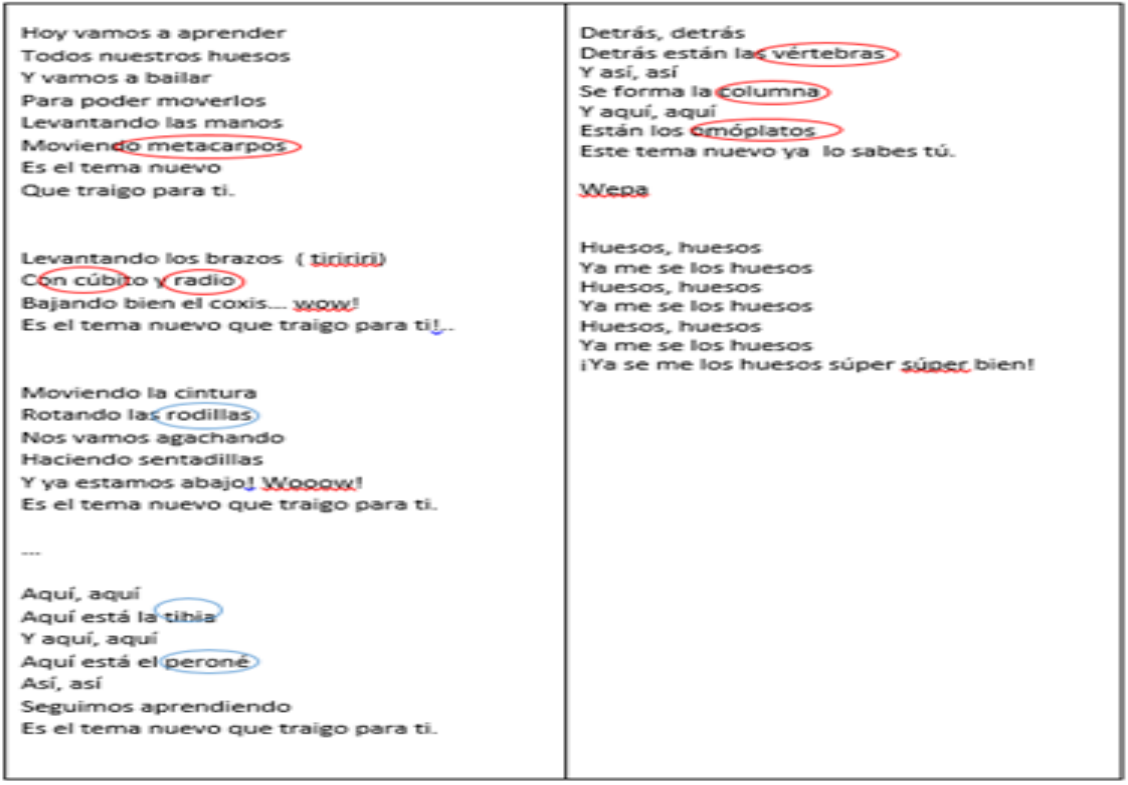

Figura 1: Letra de la canción relativa al aparato locomotor del cuerpo humano.

Con ayuda de un video (https://www.youtube.com/watch?v=5SytABu6IKY) se identifican las partes y las funciones básicas del aparato locomotor (ver Figura 2). A partir de la información del vídeo se realizó un esquema recogiendo los principales conceptos que los alumnos debían completar en sus cuadernos con lo que habían aprendido hasta el momento.

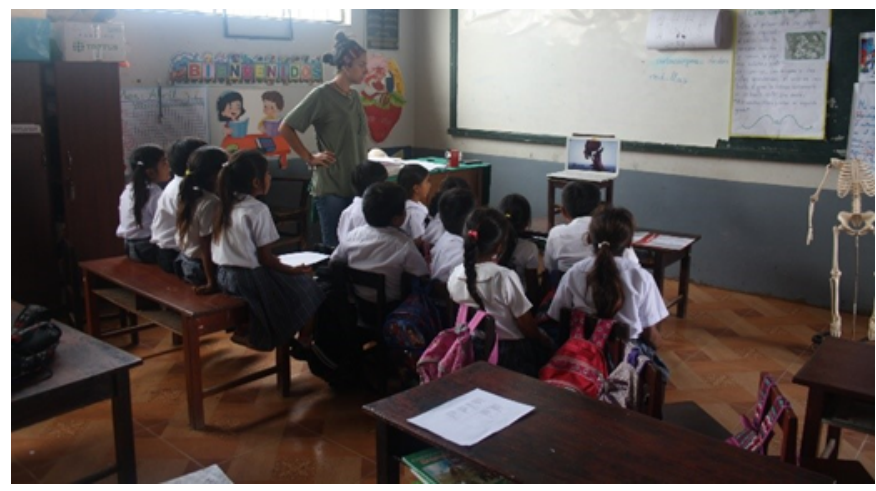

Figura 2: Visionado del vídeo sobre el aparato locomotor.

En la cuarta actividad se buscó evaluar lo que los alumnos habían aprendido. El objetivo fue que esta evaluación fuera formativa para los propios alumnos, es decir, que les sirviera como herramienta para ser conscientes de lo que habían aprendido y de lo que no. Para ello, se jugó a encontrar palabras en una sopa de letras, donde aparecía vocabulario trabajado en clase. Además, estas palabras, debían colocarlas sobre un 
dibujo del cuerpo humano. Esta actividad se desarrolló en mayor profundidad en una quinta, donde, de manera cooperativa tenían que colocar tarjetas en las que aparecían los nombres de partes del aparato locomotor, encima de un esqueleto colocado en la pizarra. Esto se trabajó a modo de juego. Una vez analizado este vocabulario de manera conceptual, se trató de desarrollar el pensamiento abstracto tan necesario en ciencias. Para ello, se trabajó sobre el propio cuerpo humano. A modo de juego, la docente iba repartiendo pegatinas y pidiendo a los alumnos que se las colocasen en la parte del aparato locomotor que iba nombrando. Los alumnos se ayudaban entre sí en caso de no reconocer o no saber ubicar ese hueso o músculo. Y, por último, la séptima actividad se diseñó para realizar una evaluación sumativa de lo trabajado en clase. En esta ocasión, se les entregó un listado de varias preguntas que debían tratar de resolver con la ayuda del material que se había ido construyendo en clase. Los resultados de estas actividades (basados en la observación y en el análisis de los materiales trabajados por los alumnos) permitieron evaluar la adecuación de la propuesta como herramienta de evaluación del aprendizaje de todos los alumnos de la clase y por tanto para evaluar si se había conseguido la inclusión de todos ellos. A continuación, se muestran los resultados recogidos en el proceso.

\section{Resultados}

En primer lugar, cabe destacar que los alumnos se han mostrado activos y participativos durante el desarrollo de las diferentes actividades. En la lluvia de ideas desarrollada como actividad inicial, a la pregunta planteada los alumnos contestaron utilizando las palabras hueso y aparato y establecieron relaciones con los huesos de los animales de los que se alimentan. Así, por ejemplo, un niño dijo: 'los huesos no nos los comemos' y otro comentó que 'un aparato es algo que sirve para que otra cosa funcione'. Sin embargo, ningún alumno utilizó las palabras músculo, ni articulación.

En el trascurso de segunda actividad, se ha observado que los alumnos estaban muy motivados. Al inicio de la canción intentaban bailarla y repetían las acciones que la docente iba realizando. Tras cantarla y bailarla se analizó de manera grupal el vocabulario que aparecía en la canción. Los resultados de este análisis mostraron que los alumnos no conocían gran parte de las palabras utilizadas. Los alumnos manifestaron que no sabían que dichas palabras eran huesos, a excepción de algunos como la columna, el cráneo o las costillas. Por lo tanto, la docente fue nombrando las palabras y aclarando si se trataban de huesos y localizando estos sobre su propio cuerpo. Por otra parte, en la lectura en común de la canción se han detectado dificultades de lectura en algunos alumnos. En estos casos, la docente iba leyendo con ellos para que pudieran sentirse participes de la clase.

A lo largo del visionado del video (tercera actividad), éste se ha ido parándo para comentar de manera conjunta lo que estaban viendo y poder facilitar el aprendizaje. Tras esto, debían realizar un esquema sobre lo que habían aprendido. La Figura 3 muestra fotos de los cuadernos personales de los alumnos donde se recogen estos resúmenes. 


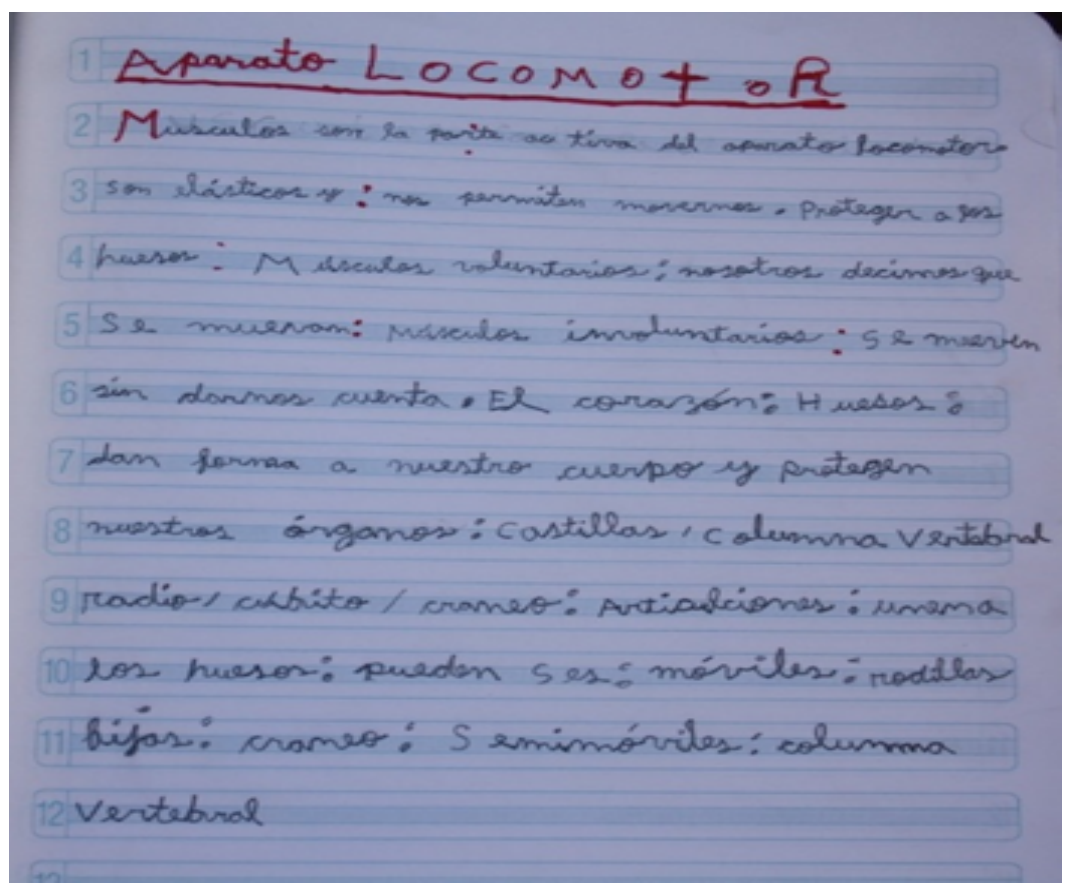

Figura 3: Resumen de la información trabajada en clase en cuaderno de uno de los alumnos.

Se puede observar como este alumno ha concluido que el aparato locomotor es el que nos permite movernos, está formado por huesos y músculos, los huesos son duros y los músculos blandos. Sin embargo, a pesar de que durante el trabajo se habían realizado comparaciones con animales que ellos conocen, esto no aparece recogido en el cuaderno del alumno. Un ejemplo de lo anterior comentado en clase fue el pollo. Este ejemplo se utilizó para identificar que lo que se come sería el músculo y lo duro el hueso. Dicha analogía les sirvió a algunas de las niñas, a las cuales les cuesta más entender los conceptos, para lograr comprenderlo.

La cuarta actividad consistió en la identificación de vocabulario específico en una sopa de letras y tras esto, debían intentar colocar cada una de las palabras sobre un esquema del cuerpo humano. Algunos alumnos completaron la actividad y otros no, los resultados se muestran en la Tabla 2.

\begin{tabular}{|c|c|c|}
\hline \multicolumn{2}{|c|}{ Tabla 2: Resultados de la sopa de letras y el cuerpo humano. } \\
\hline Alumno & Cantidad de palabras encontradas en la sopa de letras & Número de palabras colocadas correctamente en los huecos del esquema \\
\hline 1 & 8 & 6 \\
\hline 2 & 8 & 6 \\
\hline 3 & 8 & 8 \\
\hline 4 & 8 & 8 \\
\hline 5 & 8 & 8 \\
\hline 6 & 8 & 8 \\
\hline 7 & 8 & 8 \\
\hline 8 & 8 & 8 \\
\hline 9 & 8 & 8 \\
\hline 10 & 8 & 1 \\
\hline 11 & 8 & 0 \\
\hline 12 & 8 & 3 \\
\hline
\end{tabular}

Se puede observar que, en todos los casos, se han encontrado las 8 palabras en la sopa de letras. Por el contrario, a la hora de la correcta colocación de los diferentes huesos, a excepción de las tres últimas niñas, el resto de alumnos oscilan entre 8 y 6 huesos 
correctamente colocados. Aquellos alumnos que tienen 6 correctos se deben a que cúbito y radio estaban puestos en el esqueleto de manera inversa (ver Figura 4). El motivo de esto será la proximidad entre ambos huesos, y que no han sido capaces de verlo con total claridad, ni en el esqueleto, ni mientras bailábamos. Teniendo en cuenta estas tres últimas niñas, sus errores se deben a las dificultades que presentan con la lecto-escritura. En estos casos, la maestra les ha prestado ayuda leyéndoles las palabras y ellas las colocaban.

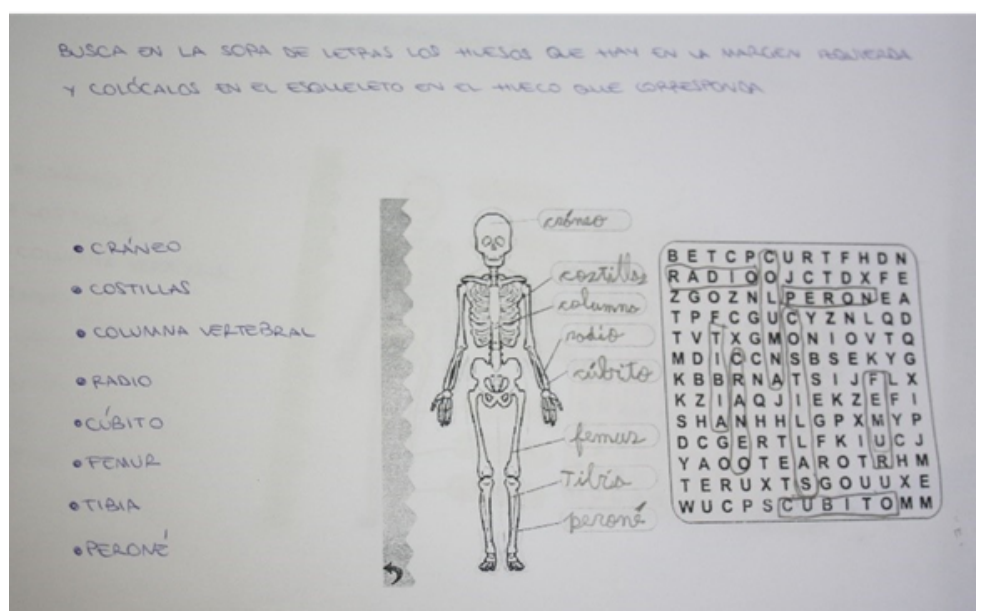

Figura 4: Ejemplo de la ficha de uno de los alumnos.

La quinta actividad se desarrolló a modo de juego. En la Figura 5 se puede observar cómo los alumnos colocaban de manera grupal el nombre de las partes del esqueleto. A las alumnas que habían manifestado problemas en la lectura se les adaptó la actividad, se les copiaba en la pizarra el nombre del hueso además de decírselo, para que así pudieran comparar la grafía y encontrar esa tarjeta.

Todos los alumnos participaron y en su mayoría colocaron bien los huesos. Se lo tomaron muy enserio e intentaban esforzarse para poner las tarjetas en el lugar adecuado, para ellos no era únicamente un juego, sino que querían realizarlo bien para que el resto de sus compañeros lo vieran.

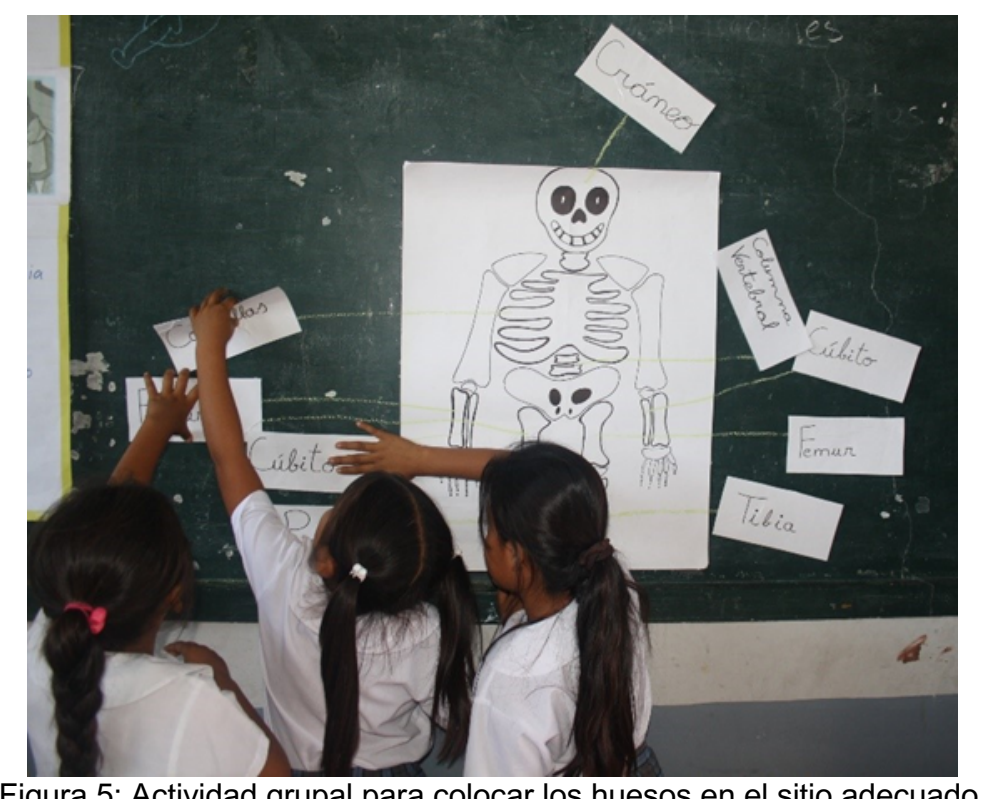

La actividad seis consistió en utilizar su propio cuerpo como medio de aprendizaje de lo trabajado en las anteriores sesiones. Para ello se fueron colocando pegatinas en las 
partes que la docente fue nombrando. Se detectaron algunos errores, por ejemplo, un caso donde una alumna colocó el cúbito en el esternón. Los alumnos se han ayudado entre sí, facilitando un aprendizaje cooperativo entre los miembros de la clase. Las niñas con problemas en la lectura no tuvieron problema en esta actividad, demostrando que conocían los términos nombrados y sabían ubicarlos.

Finalmente, los alumnos completaron unas preguntas con el objetivo de recoger el conocimiento adquirido sobre el tema trabajado (ver Figura 6).

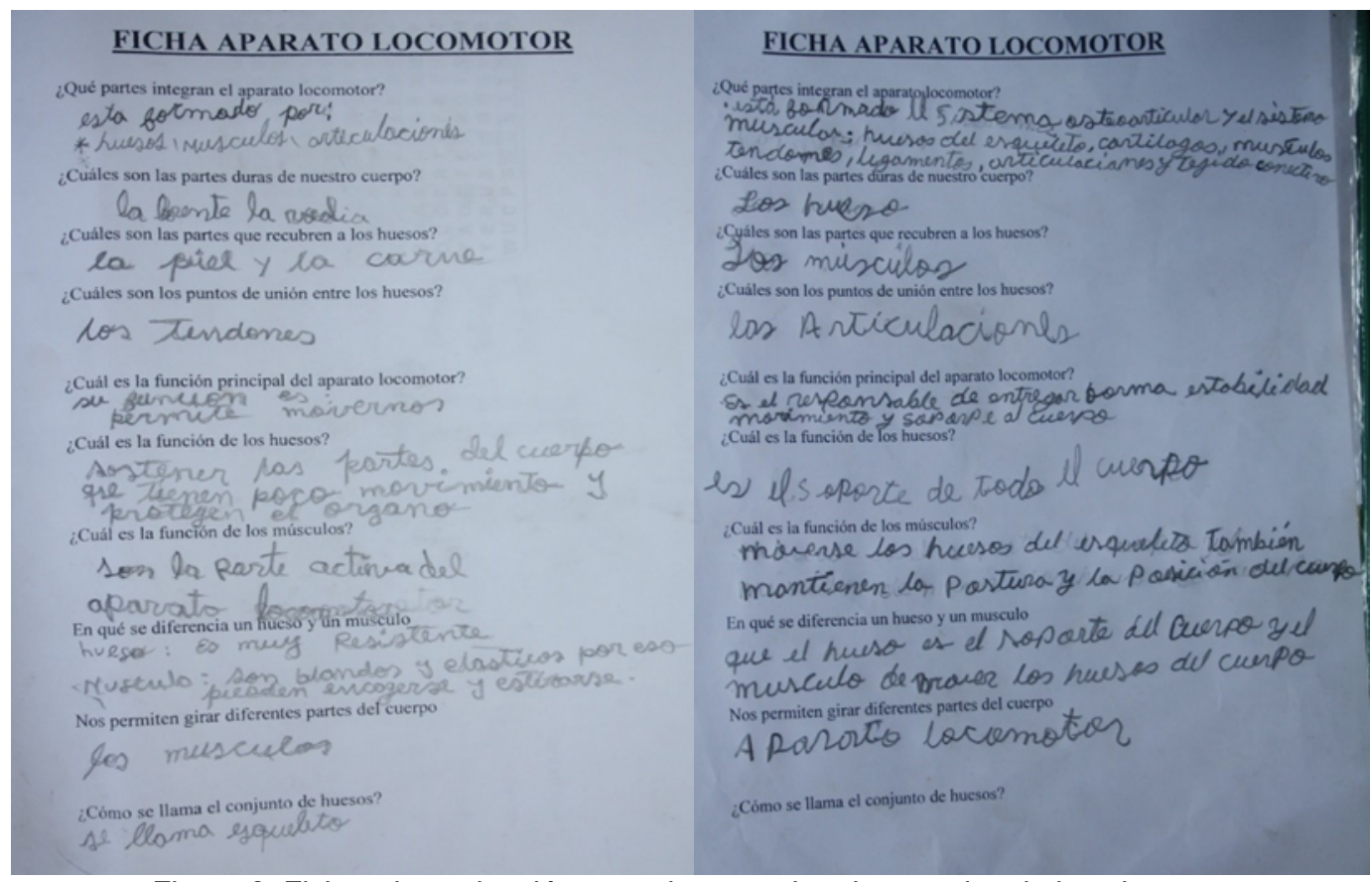

Figura 6: Fichas de evaluación sumativa completadas por dos de los alumnos.

En la Tabla 3 se analizan el número de preguntas contestadas, así como si estas son acertadas o erróneas.

\begin{tabular}{|l|c|c|c|c|}
\hline \multicolumn{5}{|c|}{ Tabla 3: Análisis de las respuestas recogidas en la ficha evaluativa de los conocimientos del alumno. } \\
\hline Pregunta & Acertadas & Erróneas & Parcialmente acertadas & No contestadas \\
\hline 1 & 5 & 4 & 2 & 1 \\
\hline 2 & 7 & 3 & 2 & 2 \\
\hline 3 & 5 & 5 & & 2 \\
\hline 4 & 4 & 6 & 1 & 3 \\
\hline 5 & 6 & 2 & 2 & 3 \\
\hline 6 & 2 & 5 & 2 & 2 \\
\hline 7 & 1 & 6 & 2 & 2 \\
\hline 8 & 2 & 6 & & 3 \\
\hline 9 & & 9 & & 4 \\
\hline 10 & 5 & 3 & & \\
\hline
\end{tabular}

La pregunta con mayor índice de respuestas acertadas son la 1, la 2, la 3 y la 5 . Por lo que se puede concluir que los alumnos han adquirido un vocabulario que desconocían (articulaciones) y han conseguido comprender que el aparato locomotor también está compuesto por músculos y articulaciones (además de los huesos). Sin embargo, sorprende que a la pregunta ¿cómo se llama el conjunto de huesos? Solo cinco alumnos hayan contestado de manera acertada, ya que este concepto se ha estado trabajando a través de todas las actividades realizadas.

La pregunta 9 no la supo contestar nadie. La mayoría de los alumnos han contestado la columna. Y la pregunta 7 les ha resultado también complicada, solo un alumno ha sido capaz de poner por escrito la función de los músculos dentro del aparato locomotor. En general, los resultados muestran que, aunque la motivación ha aumentado, se debería 
trabajar de manera más detallada cualquier contenido en ciencias. Los alumnos no están habituados a una metodología de trabajo distinta a la memorización. Aun así, la canción en la cual se habla de su cuerpo y el movimiento de éste, les ha ayudado a qe comprendan la importancia del aparato locomotor, junto con sus partes, su función principal, y que conozcan algunas partes importantes de éste.

\section{Discusión}

Tal y como se había recogido en la introducción las ciencias se deben enseñar desde edades tempranas con ejemplos cercanos a la realidad del alumno y con materiales que sean conocidos. Para que esta enseñanza se lleve a cabo de manera que todos los alumnos puedan ser incluidos en el aula, destaca la importancia de la formación del profesorado en ciencias. Esta formación no solo debe basarse en los conocimientos sobre dicha materia sino también en posibles estrategias efectivas para conseguir un aprendizaje significativo de todos y cada uno de los alumnos. Si la formación del maestro no es la adecuada, surge en él inseguridad y miedo a impartirla por lo que la metodología utilizada termina basándose en el uso de la memoria. Con una correcta formación del maestro será más fácil que los niños aprendan, ya que las actividades de motivación que realicen serán apropiadas para su edad y sus conocimientos, con lo que se sentirán más abiertos a la posibilidad de aprender algo nuevo. Así, un docente con una formación integral (conocimientos, procedimientos y actitudes) será capaz de llevar a cabo sesiones más dinámicas e interactivas, y será capaz de dar respuestas a las preguntas que planteen los alumnos.

En concreto para la enseñanza de las ciencias, se debe partir de conceptos y conocimientos cercanos al alumno para posteriormente abordar aquellos que son más complejos o que les son desconocidos, además de utilizar en ciencias materiales tangibles, de su vida cotidiana y que ellos conozcan. También será muy importante partir de hechos que les preocupen para después abordarlos desde la visión experimental y científica, logrando llegar así a la base teórica. Conforme se va avanzando, en los diferentes cursos, debe aumentar la complejidad y la cantidad de conceptos a abordar.

En el presente trabajo se ha indagado sobre cómo, al trabajar una propuesta de manera interdisciplinar, en grupo cooperativo y de manera práctica, los alumnos han alcanzado los aprendizajes esperados. Los alumnos de la clase donde se ha desarrollado la propuesta estaban habituados a trabajar por medio de la repetición para conseguir la memorización de los conceptos. No se trabajaban procedimientos ni actitudes hacia la ciencia. No se conseguía tampoco la inclusión efectiva de tres alumnas con necesidades especiales debidas a sus dificultades de aprendizaje. Al cambiar el tipo de metodología docente, adaptada al alumnado, mediante la cual el alumno protagoniza su aprendizaje se mostraron nerviosos por la falta de costumbre. Sin embargo, a lo largo de la primera sesión, la motivación de los alumnos aumentó, pidiendo participar y contestar a las preguntas que se formulaban en clase. También el uso de otras materias, como la música, para trabajar el tema del aparato locomotor ha resultado un buen instrumento motivacional. Por otra parte, a los alumnos les encanta salir de la rutina y realizar actividades nuevas, cómo por ejemplo juegos para conocer los nombres de los huesos y su ubicación en el cuerpo humano. Finalmente, incluso aquellas niñas que presentan dificultades de lecto-escritura lograron conocer el nombre de algunos huesos y conocer dónde se encuentran en su propio cuerpo. Para ello, se adaptaron todas las actividades en las que podían encontrar dificultades, permitiendo esto un aprendizaje inclusivo para estas alumnas.

Se puede concluir también que a pesar de que los alumnos han estado mucho más motivados, trabajar puntualmente de manera práctica no asegura la adquisición de 
conocimientos. Cuando los alumnos están habituados a trabajar de manera memorística, hace falta tiempo para lograr que desarrollen sus competencias con otras metodologías. Sin embargo, si el objetivo es que todos los alumnos estén incluidos y contemplados en el proceso de enseñanza, debe hacerse el esfuerzo.

\section{Referencias}

Altun, F. y Hikmet, Y. (2010). Learning styles of the gifted students in Turkey. Procedia Social and Behavioral Sciences, 9, 198202.

Barmby, P., Kind. P.M. y Jones, K. (2008). Examining changing attitudes in secondary school science. International Journal of Science Education, 30(8), 1075-1093.

Calvo, M.I., Verdugo, M.A. y Amor, A.M. (2016). La participación familiar es un requisito imprescindible para una escuela inclusiva. Revista Latinoamericana de Educación Inclusiva, 10(1), 99-113.

Cascarosa, E. y Marco, M. (2018). Las vocaciones científicas en Educación Primaria. Una investigación inicial en Perú y España. Reidocrea, 7(17), 190-201.

Cortés, A.L., de la Gándara, M., Calvo, J.M., Ibarra, J., Arlegui, J., Martínez, M.B. y Gil, M. J. (2012). Expectativas, necesidades y oportunidades de los maestros en formación ante la enseñanza de las ciencias en la Educación Primaria. Enseñanza de las Ciencias, 30, 155-176.

Cuevas, P., Lee, O., Hart, J. y Deakort, R. (2005). Improving science inquiry with elementary students of diverse backgrounds. Journal of Research in Science Teaching, 42, 337-357. http://dx.doi.org/10.1002/tea.20053

Díaz, H. (2015). Formación docente en el Perú, realidades y tendencias. Santillana. ISBN: 978-612-01-0221-3

Gallegos, C. (2001). El currículo de primaria basado en actividades como puente entre la cultura local y global: una experiencia educativa en la Amazonía Peruana. Cultura y Educación, 13:1, 73-92,

García, S. y Martínez, C. (2001). Qué actividades y qué procedimientos utiliza y valora en profesorado en Educación Primaria. Enseñanza de las Ciencias, 19(3), 433-452.

George, R. (2006). A cross-domain analysis of change in students' attitudes toward science and attitudes about the utility of science. International Journal of Science Education, 28(6), $571-589$.

Gil-Flores, J. (2012). Actitudes del alumnado español hacia las ciencias en la evaluación PISA 2006. Enseñanza de las Ciencias, 30(2), 131-152.

Harlen, W. (2015). Working with Big ideas of Science Education. Trieste (Italia): Science Education Programme of IAP.

Izquierdo, M., Sanmartí, N. y Espinet, M. (1999). Fundamentación y diseño de las prácticas escolares de ciencias experimentales. Enseñanza de las Ciencias, 17(1). 45-59.

Kozulín, A. (2000). Instrumentos psicológicos, en Barcelona: Paidós

Lena, P. (2011). International evidence shows teacher preparation is vital, en E. Yeomans (ed.). Perspective on Education: Inquiry-Based Learning 8-11. London: Welcome Trust.

Marín, V., Negre, F. y Pérez, A. (2014). Entornos y redes personales de aprendizaje (PLE-PLN) para el aprendizaje colaborativo. Comunicar, 42, 35-43.
Solbes, J., Montserrat R. y Furió, C. (2007). El desinterés del alumnado hacia el aprendizaje de la ciencia: implicaciones en su enseñanza. Didáctica de las Ciencias Experimentales y Sociales, 21, 91-117.

Tanggaard, L. (2014). A Situated Model of Creative Learning. European Educational Research Journal, 13(1), 107-116. 